\title{
Propofol protects against oxygen/glucose deprivation-induced cell injury via gap junction inhibition in astrocytes
}

\author{
YANTING FAN $^{1 *}$, SIYU ZHU $^{2 *}$, JING WANG $^{3}$, YUPING ZHAO $^{1}$ and XUDONG WANG ${ }^{3}$ \\ ${ }^{1}$ Department of Anesthesiology, Guangzhou Women and Children's Medical Center, \\ Guangzhou Medical University, Guangzhou, Guangdong 510623; ${ }^{2}$ Department of Medical Imaging, \\ Collaborative Innovation Center for Cancer Medicine, State Key Laboratory of Oncology in South China, \\ Sun Yat-sen University Cancer Center; ${ }^{3}$ Department of Anesthesiology, State Key Laboratory of Oncology \\ in South China, Sun Yat-sen University Cancer Center, Guangzhou, Guangdong 510060, P.R. China
}

Received February 6, 2020; Accepted June 22, 2020

DOI: $10.3892 / \mathrm{mmr} .2020 .11357$

\begin{abstract}
Stroke is one of the leading causes of mortality and disability worldwide with limited clinical therapies available. The present study isolated primary astrocytes from the brains of rats and treated them with oxygen-glucose deprivation and re-oxygenation (OGD/R) to mimic hypoxia/reperfusion (H/R) injury in vitro to investigate stroke. It was revealed that propofol (2,6-diisopropylphenol), an intravenous sedative and anesthetic agent, protected against oxygen/glucose-deprivation (OGD) and induced cell injury. Furthermore, propofol exerted a protective effect by inhibiting gap junction function, which was also revealed to promote cell death in astrocytes. The present study further identified that propofol suppressed gap junction function by downregulating the protein expression levels of connexin43 (Cx43), which is one of the most essential components of gap junctions in astrocytes. In addition, when the expression levels of $\mathrm{Cx} 43$ were downregulated using small interfering RNA, OGD/R-induced cell death was decreased. Conversely, cell death was enhanced when $\mathrm{Cx} 43$ was overexpressed, which was reversed following propofol treatment. In summary, propofol protects against OGD-induced injury in astrocytes by decreasing the protein expression levels of Cx43 and suppressing gap junction function. The present study improved our understanding of how propofol protects astrocytes from OGD/R-induced injury.
\end{abstract}

Correspondence to: Dr Xudong Wang, Department of Anesthesiology, State Key Laboratory of Oncology in South China, Sun Yat-sen University Cancer Center, 651 East Dongfeng Road, Guangzhou, Guangdong 510060, P.R. China

E-mail: wangxd@sysucc.org.cn

*Contributed equally

Key words: oxygen-glucose deprivation and re-oxygenation, astrocytes, propofol, gap junction, connexin 43

\section{Introduction}

Stroke is the second leading cause of mortality and the third leading cause of disability worldwide (1). In the past two decades, both the morbidity and mortality rates of stroke have markedly increased in China, which leads to substantial health care expenditures (2). This is a result of limited treatment options for stroke. Therefore, identifying novel drugs to treat stroke and identifying its molecular mechanism is of great significance.

Astrocytes are the most abundant glial cells in the central nervous system. Astrocytes have been reported to serve important roles in protecting neurons after stroke in multiple ways, including antioxidant activities, ion homeostasis, energy transfer and neurotransmitter transport (3). Furthermore, it has been reported that impaired astrocytes in stroke exacerbate neuronal death (4). Therefore, alleviating astrocyte injury is a promising direction to prevent excessive neuronal damage following stroke.

Gap junctions are intercellular membrane channels that directly connect the cytoplasm of adjacent cells (5). A gap junction channel contains two hemichannels, each of which consists of six connexin (Cx) monomers (5). Intercellular communication via gap junctions serves a vital role in the development of oxygen-glucose deprivation and re-oxygenation (OGD/R) injury, which is a leading cause of stoke (6). Cx43 is one of the most abundant gap junction proteins in astrocytes (7). However, the function of Cx43 in hypoxia/reperfusion (H/R) injury remains debatable. For instance, Sun et al (8) identified that upregulation of $\mathrm{Cx} 43$ expression could inhibit oxidative damage and subsequently decrease apoptotic cell death in cerebral ischemic injury. In addition, Cx43 knockout could increase infarct volumes in mice subjected to transient middle cerebral artery occlusion (9). However, other studies have demonstrated that $\mathrm{Cx} 43$ aggravates ischemia-induced cell damage by spreading death signals to neighboring cells $(10,11)$. Therefore, the role of gap junctions and $\mathrm{Cx} 43$ in stroke development remains unclear.

Propofol is widely used in anesthesia and intensive care units for both short-term anesthesia and longer-term sedation (12). Previous studies have found that propofol exerts 
protective effects in models of cerebral H/R injury (13-16). However, the underlying molecular mechanisms of the neuroprotective effects of propofol against cerebral ischemia remain unclear. The present study investigated the effect of propofol on cerebral ischemic injury and its roles in regulating gap junction function.

\section{Materials and methods}

Primary culture of astrocytes. The animal experiment protocol was reviewed and approved by the Institutional Animal Care and Use Committee of Sun Yat-sen University (Guangzhou, China). A total of 40 Sprague-Dawley rats (age, 1-2 days; weight, 5-7 g; male) were obtained from Sun Yat-sen University. The animals were housed at $22-24^{\circ} \mathrm{C}$ and $55 \pm 5 \%$ relative humidity, with a 12-h light/dark cycle and free access to food and water. For each primary culture, 6-10 rats were used to isolate cortical astrocytes as previously described $(17,18)$. Specifically, the brain was excised from these rats and the cerebral cortices were minced with forceps and harvested. The cortical chunks were placed in $\mathrm{Ca}^{2+}$-free phosphate buffered saline (PBS) containing 0.5\% trypsin and $5 \mathrm{mM}$ EDTA, and digested at $37^{\circ} \mathrm{C}$ for $20 \mathrm{~min}$. Subsequently, the dissociated cells were centrifuged, resuspended in Dulbecco's modified Eagle's medium/F12 (DMEM/F12) containing 10\% fetal bovine serum (FBS), $100 \mathrm{U} / \mathrm{ml}$ penicillin and $100 \mu \mathrm{g} / \mathrm{ml}$ streptomycin (all cell culture reagents were purchased from Gibco; Thermo Fisher Scientific, Inc.), and seeded into poly-1-lysine-coated flasks at a density of $1 \times 10^{5}$ cells $/ \mathrm{cm}^{2}$. The culture medium was refreshed every 2 days. Upon reaching confluence (day 8-10 of culture), the flasks were rotated at $200 \mathrm{rpm}$ for $16 \mathrm{~h}$ at $37^{\circ} \mathrm{C}$ on an orbital shaker to remove microglia and oligodendrocytes. The astrocytes that attached to the bottom of the flasks were trypsinized, resuspended and seeded into cell culture plates.

Immunocytochemistry. Astrocyte purity was assessed using immunocytochemical staining of glial fibrillary acidic protein (GFAP). Briefly, $1 \times 10^{5}$ astrocytes $/ \mathrm{cm}^{2}$ were seeded onto coverslips, grown for $48 \mathrm{~h}$ and fixed with $4 \%(\mathrm{w} / \mathrm{v})$ paraformaldehyde at room temperature for $30 \mathrm{~min}$. The astrocytes were then incubated in PBS containing 5\% (w/v) sheep serum (Sigma-Aldrich; Merck KGaA) and 0.3\% (w/v) Triton X-100 (Sigma-Aldrich; Merck KGaA) at room temperature for $20 \mathrm{~min}$. The astrocytes were then transferred to $2 \%$ bovine serum albumin (Sigma-Aldrich; Merck KGaA) containing anti-GFAP antibody (1:500; cat. no. AB5804; Sigma-Aldrich; Merck KGaA) and incubated at $4^{\circ} \mathrm{C}$ overnight. The next day, the coverslips were washed with PBS and incubated with a Cy3-conjugated secondary antibody (1:500; cat. no. A10520; Invitrogen; Thermo Fisher Scientific, Inc.) for $1 \mathrm{~h}$ at room temperature. Nuclear counterstaining was performed using DAPI (1:1,000; cat. no. D9542; Sigma-Aldrich; Merck KGaA) at room temperature for $5 \mathrm{~min}$. The coverslips were reviewed and scored by a pathologist under a fluorescence microscope (magnification, x40; Olympus IX71; Olympus Corporation). Results are presented as the number of cells marked with GFAP/the total number of nuclei counterstained.

Drug treatment. Pure propofol (2,6-diisopropylphenol; Sigma-Aldrich; Merck KGaA) was dissolved in intra-lipid
(10\% soybean oil, $2.25 \%$ glycerol, $1.2 \%$ purified egg phosphatide; Clintec International SARL) prior to use, at a final concentration of 10,20 or $40 \mu \mathrm{M}$ in the culture medium. The control contained the same amount of intra-lipid only. Carbenoxolone (CBX; Sigma-Aldrich; Merck KGaA) was dissolved in PBS to give $50 \mathrm{mM}$ stock solution. The final concentration of CBX used was $50 \mu \mathrm{M}$, which was determined according to a previous study (19). For the experiments, propofol, intra-lipid and/or CBX were added to the culture medium, and astrocytes were cultured for $1 \mathrm{~h}$ before OGD exposure. The cultured primary astrocytes were randomly assigned to different drug treatments.

OGD/R treatment of astrocytes. After the drug treatment, the astrocyte culture was washed twice with glucose-free PBS, and glucose-free DMEM/F12 was added. Subsequently, the culture plates were placed in an airtight chamber pumped with a hypoxic gas mixture $\left(95 \% \mathrm{~N}_{2}, 5 \% \mathrm{CO}_{2}\right.$; Advanced ${ }^{\circledR}$ Anoxomat; Advanced Instruments Inc.) at $37^{\circ} \mathrm{C}$ and exposed to OGD for $6 \mathrm{~h}$. Then, the medium was changed to regular DMEM/F12 with $10 \% \mathrm{FBS}$ and reoxygenated in a water-saturated atmosphere of $5 \% \mathrm{CO}_{2}$ and $95 \%$ air for $24 \mathrm{~h}$ in order to mimic a reperfusion period. Astrocytes that did not undergo any treatment were defined as control cells. Astrocytes in the sham cell group were exposed to $6 \mathrm{~h} \mathrm{OGD} / 24 \mathrm{~h}$ reoxygenation. Astrocytes in the lipid group were pretreated with intra-lipid for $1 \mathrm{~h}$ before exposure to $\mathrm{OGD} / \mathrm{R}$.

Cell viability and lactate dehydrogenase (LDH) secretion. Cell viability and LDH secretion assays were performed to examine astrocyte damage as previously described (20). Astrocyte viability was assessed using a Cell Counting Kit-8 (CCK-8) assay, according to the manufacturer's protocol (Dojindo Molecular Technologies, Inc.). This kit was used to monitor the survival and proliferation of cells. In the present

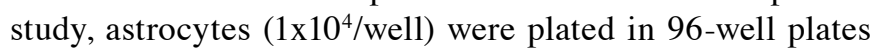
and incubated for $24 \mathrm{~h}$ to achieve complete cell adhesion. After exposure to different experimental conditions, as aforementioned, cell cultures were washed with DMEM/F12 and $10 \mu 1$ 10\% CCK-8 solution was added into each well. The cell were cultured for another $2 \mathrm{~h}$. Cell viability was measured as absorbance at an optical density of $450 \mathrm{~nm}$ using an Epoch ${ }^{\mathrm{TM}}$ microplate reader (BioTek Instruments, Inc.). Each assay was performed in triplicate. Data were summarized as a percentage of the control.

In addition, an LDH-Cytotoxicity assay kit (cat. no. 11644793001; Sigma-Aldrich; Merck KGaA) was used to assess LDH activity, according to the manufacturer's protocol. The culture media of astrocytes were collected after exposure to $\mathrm{OGD} / \mathrm{R}$ and then centrifuged at $100 \mathrm{x} \mathrm{g}$ for $5 \mathrm{~min}$ at room temperature to obtain the supernatant. Subsequently, the astrocytes were lysed using 1\% Triton X-100 (Sigma Aldrich; Merck KGaA). Then, $50 \mu \mathrm{l}$ culture supernatant or cell lysates were incubated with $\mathrm{LDH}$ reaction mixture at $37^{\circ} \mathrm{C}$ for $20 \mathrm{~min}$. The intensity of color was measured at $490 \mathrm{~nm}$ using a spectrophotometer. Data were summarized as a percentage of total $\mathrm{LDH}$.

'Parachute' dye-coupling assay. Gap junction function was assessed, as previously described $(21,22)$. Briefly, cells were 
cultured to confluence, and donor cells were labelled with $5 \mu \mathrm{mol} / \mathrm{l}$ calcein-acetoxymethyl ester (Invitrogen; Thermo Fisher Scientific, Inc.), which can permeate through gap junctions to adjacent cells. After cells were incubated for $30 \mathrm{~min}$ at $37^{\circ} \mathrm{C}$, the donor cells were trypsinized and seeded onto the receiver cells at a 1:150 donor/receiver ratio. These two types of cells were allowed to attach to form gap junctions at $37^{\circ} \mathrm{C}$ for $4 \mathrm{~h}$, and were investigated under a fluorescence microscope (magnification, x40; Olympus IX71; Olympus Corporation). The average number of receiver cells containing dye per donor cell was evaluated and normalized to that of control cultures as a measurement of gap junction intercellular communication (GJIC) levels. The number of receiver cells for each condition were counted by the same observer who was blinded to the treatment conditions.

Flow cytometry apoptosis assay. Cell apoptosis was detected using an Alexa Fluor ${ }^{\circledR} 488$ Annexin V/propidium iodide (PI) staining kit (Invitrogen; Thermo Fisher Scientific, Inc.). Cells from the different experimental groups were harvested, washed in ice-cold PBS, centrifuged at $100 \mathrm{x} g$ for $5 \mathrm{~min}$ at $4^{\circ} \mathrm{C}$, re-centrifuged using the same conditions, and resuspended in $400 \mu 1 \mathrm{1X}$ Annexin-binding buffer. Annexin V/PI staining solution was added to the mixture and cells were incubated in the dark for $15 \mathrm{~min}$. Afterwards, $400 \mu \mathrm{l}$ $1 \mathrm{X}$ Annexin-binding buffer was added to these mixtures and mixed gently. The green fluorescence of Annexin V (FL1) vs. red fluorescence of PI (FL2) was analyzed using a Beckman CytoFLEX flow cytometer (Beckman Coulter, Inc.) at 530 and $575 \mathrm{~nm}$, respectively. At least $2 \times 10^{4}$ cells were examined per sample. Early apoptotic cells bound to Annexin V, but not to PI, whereas late apoptotic/necrotic cells displayed both types of binding, which was analyzed using WinMDI 2.8 software (The Scripps Research Institute).

Protein extraction and western blotting. Astrocytes in different treatment groups were washed three times with ice-cold PBS and lysed with RIPA lysis buffer (Bio-Rad, Laboratories, Inc.) and protease inhibitors (1:200) on ice for $15 \mathrm{~min}$. Then, cells were sonicated on ice-cold water with 2-3 cycles of sonication for 15 and $45 \mathrm{sec}$ of cooling time and then centrifuged at $16,000 \mathrm{xg}$ for $30 \mathrm{~min}$ at $4^{\circ} \mathrm{C}$. Protein concentration was determined by a bicinchoninic acid protein assay using a UV spectrophotometer (DU 640; Beckman Coulter, Inc.). The protein samples $(25 \mu \mathrm{g})$ were separated by $10 \%$ SDS-PAGE, transferred onto PVDF membranes and blocked with 5\% fat-free milk for $1 \mathrm{~h}$ at room temperature. Subsequently, the membranes were incubated at $4^{\circ} \mathrm{C}$ overnight with an anti-Cx43 primary antibody (1:1,000; cat. no. SAB4501175; Sigma-Aldrich; Merck KGaA). Following the primary antibody incubation, the membrane was washed with TBS $+0.1 \%$ Tween-20 (TBST) three times at room temperature and incubated for $1 \mathrm{~h}$ at room temperature with a secondary antibody (1:4,000; cat. no. RABHRP1; Sigma-Aldrich; Merck KGaA). The membrane was then washed with TBST five times at room temperature and visualized using an ECL reagent (Beyotime Institute of Biotechnology) and an ECL detection system (Beyotime Institute of Biotechnology). An anti-GAPDH antibody (1:10,000; cat. no. G9545; Sigma-Aldrich; Merck
$\mathrm{KGaA}$ ) was used to confirm equal loading. The protein bands were analyzed using Quantity One 1-D v4.52 software and a GS-800 densitometer (Bio-Rad, Laboratories, Inc.).

Knockdown and overexpression of Cx43. In order to knockdown $\mathrm{Cx} 43$ expression in astrocytes $\left(1 \times 10^{5}\right.$ cells $\left./ \mathrm{cm}^{2}\right)$, a synthetic small interfering RNA (siRNA; 100 pmol) from Guangzhou RiboBio Co., Ltd. was transiently transfected into astrocytes for $48 \mathrm{~h}$ using Lipofectamine ${ }^{\circledR} 2000$ (Invitrogen; Thermo Fisher Scientific, Inc.). The target sequence of the siRNA was: 5'-GCTGGTTACTGGTGA CAGA-3'. Non-targeting siRNA (5'-UUCUCCGAACGU GUCACGU-3'; 100 pmol) was used as a negative control. Overexpression of $\mathrm{Cx} 43$ was achieved by transient transfection of $2 \mu \mathrm{g}$ pcDNA3.1-Cx43 (Addgene, Inc.) using $3 \mu \mathrm{l}$ Lipofectamine 2000. An empty vector was transfected $(2 \mu \mathrm{g})$ as the negative control. Following $48 \mathrm{~h}$ of transfection at $37^{\circ} \mathrm{C}$, the knockdown and overexpression efficiency were assessed by western blotting.

Statistical analysis. Data were statistically analyzed using SPSS 15.0 software (SPSS, Inc.) and are presented as the mean \pm SEM. For each treatment group, three repeats $(n=3)$ were performed, and experiments were performed according to our previous study (23). Multiple comparisons among groups were analyzed by one-way ANOVA with Tukey's post hoc test. All analyses were plotted using SigmaPlot 13.0 (Systat Software, Inc.). P $<0.05$ was considered to indicate a statistically significant difference.

\section{Results}

OGD/R-induced cell injury is gap junction-dependent. To study OGD/R-induced cell injury, cortical astrocytes were isolated from rats and their purity was verified by immunostaining. Immunofluorescence staining for GFAP was used to label astrocytes, whereas DAPI was used to label cell nuclei (Fig. 1A). The purity of isolated astrocytes was $>98 \%$ (data not shown). First, the gap junction inhibitor CBX was used to study the function of gap junctions in OGD/R-induced cell injury. Astrocytes were pretreated with $50 \mu \mathrm{M} \mathrm{CBX}$ for $1 \mathrm{~h}$, followed by hypoxia treatment for $6 \mathrm{~h}$ and reperfusion for $24 \mathrm{~h}$. The inhibitory effect of CBX on gap junction was verified by a 'parachute' dye-coupling assay. The data demonstrated that $50 \mu \mathrm{M} \mathrm{CBX}$ pretreatment for $1 \mathrm{~h}$ could markedly decrease GJIC (Fig. 1B).

Subsequently, to study the role of gap junctions in OGD/R-induced cell injury, the cell viability, LDH leakage rate and apoptotic rate were investigated using a WST-8 Cell Counting Kit, LDH cytotoxicity assay kit and flow cytometry, respectively. It was revealed that $\mathrm{OGD} / \mathrm{R}$ treatment markedly decreased the viability of astrocytes, and increased LDH leakage and cell apoptotic rates, whereas CBX itself did not affect cell viability. In the OGD/R-treated group that were pretreated with CBX, the cell viability rate was increased by $\sim 20 \%$, the LDH leakage rate was decreased by $\sim 10 \%$, and the apoptotic rate was decreased by $\sim 15 \%$ compared with those in the OGD/R-treated group without CBX pretreatment (Fig. 1C-E). Collectively, these data indicated that the gap junction promoted OGD/R-induced cell injury in astrocytes. 
A

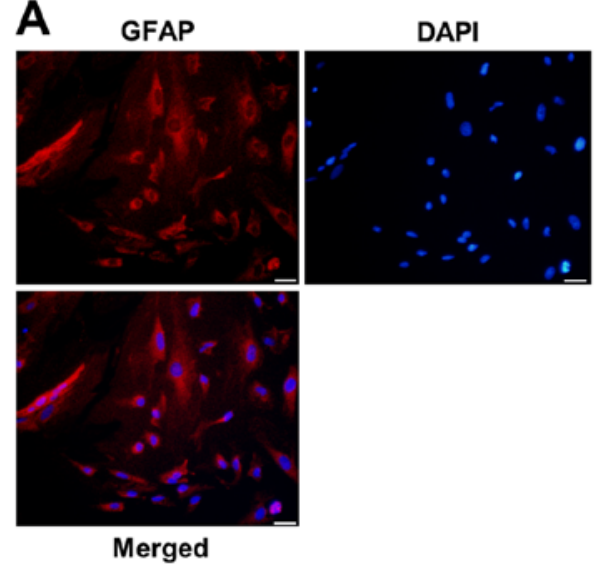

B
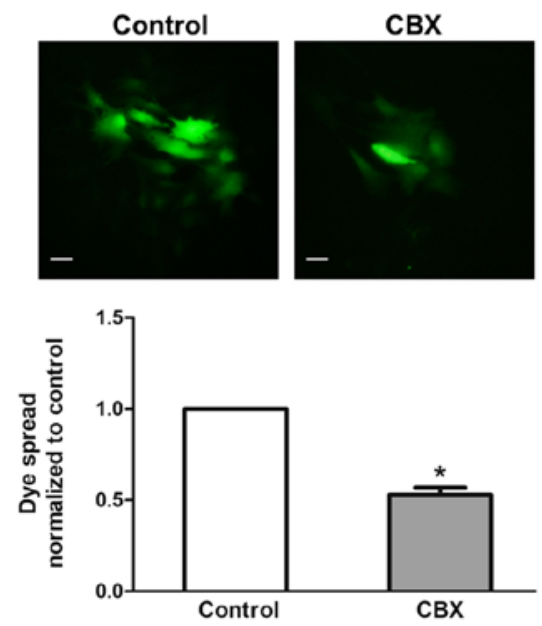

C

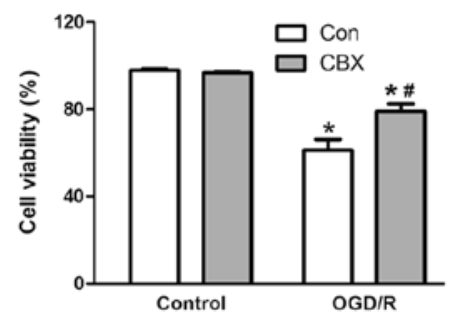

D

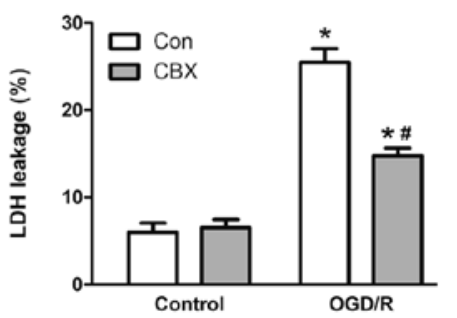

E

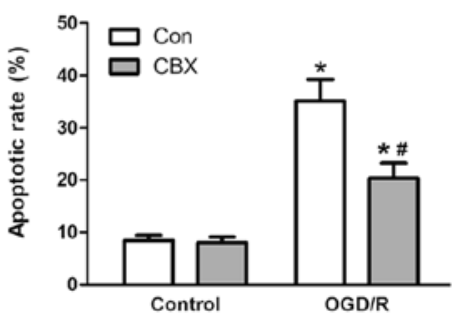

$\mathbf{F}$

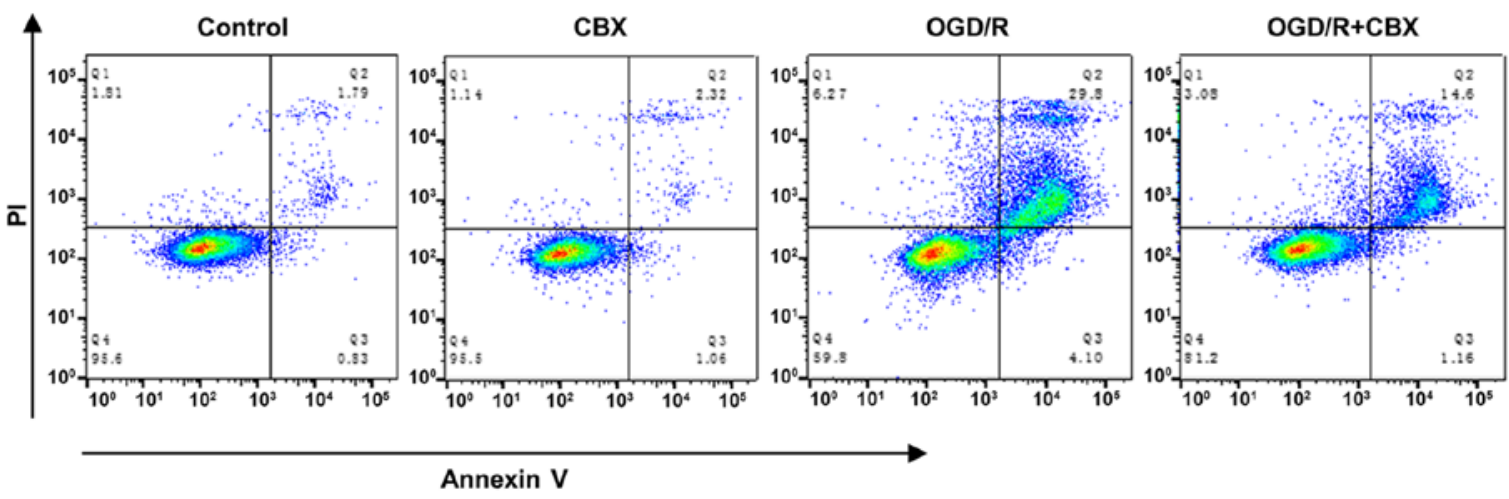

Figure 1. Effect of gap junction function on OGD/R-induced cell injury. (A) To confirm the establishment of a primary culture of the astrocyte OGD injury model, primary cultured astrocytes were labeled with anti-GFAP and counterstained with DAPI nuclear stain. Scale bar, 20- $\mu$ m. (B) Effect of GJIC inhibitor $\mathrm{CBX}(50 \mu \mathrm{M})$ on dye coupling. Scale bar, $20-\mu \mathrm{m}$. (C) Astrocyte injury following exposure to OGD/R in terms of cell viability, which was assessed using a Cell Counting Kit-8 assay, and (D) LDH release rate, assessed by the LDH-cytotoxicity assay. (E) Apoptotic rate measured by flow cytometry. (F) Representative flow cytometry images. The percentage of apoptotic cells includes cells that were Annexin $\mathrm{V}^{+}$and $\mathrm{Annexin} \mathrm{V}^{+} / \mathrm{PI}^{+}$after $\mathrm{H} / \mathrm{R}$. Values are presented as the mean \pm SEM of three independent experiments. ${ }^{*} \mathrm{P}<0.05$ vs. control group without $\mathrm{OGD} / \mathrm{R}$ treatment; ${ }^{\#} \mathrm{P}<0.05$ vs. OGD/R-treated group without $\mathrm{CBX}$ pretreatment. CBX, carbenoxolone; GFAP, glial fibrillary acidic protein; GJIC, gap junction intercellular communication; H/R, hypoxia/reoxygenation; LDH, lactate dehydrogenase; OGD/R, oxygen-glucose deprivation and re-oxygenation; PI, propidium iodide.

Propofol protects astrocytes from OGD/R-induced cell injury. To investigate the effect of propofol on OGD/R-induced cell injury, astrocytes were pretreated with 10,20 and $40 \mu \mathrm{M}$ propofol for $1 \mathrm{~h}$ followed by OGD/R treatment. The cell viability, LDH leakage rate and cell apoptotic rate were examined. It was demonstrated that propofol (starting from $20 \mu \mathrm{M}$ ) increased cell viability, and reduced LDH leakage and the cell apoptosis rate compared with the sham group (OGD/R-treated without propofol pretreatment) (Fig. 2A-D), indicating that propofol protected astrocytes from OGD/R-induced cell injury.

Propofol inhibits gap junction function and decreases Cx43 expression in astrocytes. Since both propofol and gap junction inhibitor CBX protected astrocytes from OGD/R-induced injury, to further study whether propofol exerted protective effects by inhibiting gap junction function, the present study tested the effect of propofol on gap junction function by dye coupling between astrocytes. Propofol (10, 20 and $40 \mu \mathrm{M})$ treatment for $1 \mathrm{~h}$ suppressed dye spread in astrocytes in a dose-dependent manner when compared with control group (Fig. 3A), indicating that propofol inhibited gap junction function on astrocytes.

Gap junction function is mediated by the protein levels of a variety of $\mathrm{Cx}$ proteins. $\mathrm{Cx} 43$ is one of the most abundant $\mathrm{Cx}$ proteins in astrocytes (7). Furthermore, Cx43 has a neuroprotective effect in cerebral ischemic injury (8). Therefore, it was 

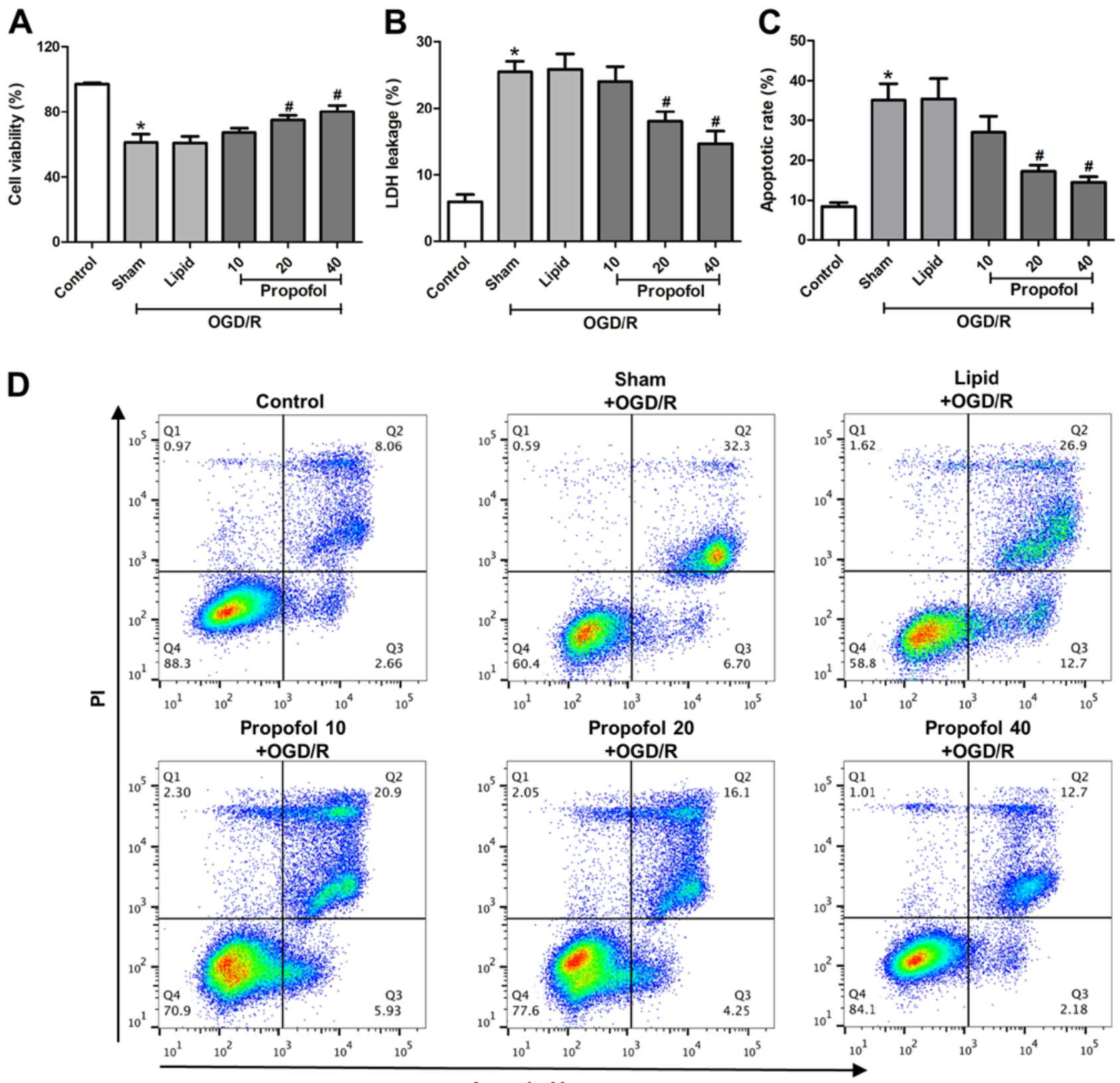

Annexin V

Figure 2. Effect of propofol on OGD/R-induced cell injury in astrocytes. Astrocytes were pretreated with propofol (10,20 and $40 \mu \mathrm{M})$ for $1 \mathrm{~h}$ prior to OGD/R treatment. (A) Cell viability, (B) LDH release and (C) apoptotic rate. (D) Representative flow cytometry images. Values are presented as the mean \pm SEM of three independent experiments. ${ }^{\mathrm{P}}<0.05$ vs. control group; ${ }^{*} \mathrm{P}<0.05$ vs. sham group (cells exposed to OGD/R injury without propofol pretreatment). $\mathrm{LDH}$, lactate dehydrogenase; OGD/R, oxygen-glucose deprivation and re-oxygenation.

investigated whether propofol inhibited gap junction function via $\mathrm{Cx} 43$. Astrocytes were treated with different concentrations of propofol $(10,20$ and $40 \mu \mathrm{M})$ for $1 \mathrm{~h}$ and the protein levels of $\mathrm{Cx} 43$ were measured. Propofol significantly decreased the protein levels of $\mathrm{Cx} 43$ in a dose-dependent manner (Fig. 3B). These data suggested that propofol inhibited gap junction function by downregulating the expression levels of $\mathrm{Cx} 43$ in astrocytes.

Neuroprotective effect of propofol against $O G D / R$ is dependent on gap junctions and $C x 43$. To further validate the role of $\mathrm{Cx} 43$ in propofol-mediated neuroprotective effects in injured astrocytes, $\mathrm{Cx} 43$ was overexpressed or knocked down. As shown in Fig. 4A, the expression levels of Cx43 were upregulated in the Cx43 overexpression group (Cx43 $\mathrm{OE})$, whereas expression was markedly downregulated in the Cx43 knockdown group (Cx43 KD) compared with the control group. First, the effect of $\mathrm{Cx} 43 \mathrm{OE}$ and KD on gap junction function was investigated. As expected, $\mathrm{Cx} 43 \mathrm{OE}$ significantly promoted gap junction function, whereas $\mathrm{Cx} 43$ $\mathrm{KD}$ inhibited it, when measuring the dye coupling in astrocytes (Fig. 4B).

Subsequently, the effects of propofol on Cx43 OE and KD cells were studied. The cell viability of the Cx43 OE group was significantly decreased after OGD/R injury compared with that in the normal $\mathrm{Cx} 43$ group, whereas the apoptotic rate in the $\mathrm{Cx} 43 \mathrm{OE}$ group was significantly increased. These data indicated that cells with higher expression levels of Cx43 

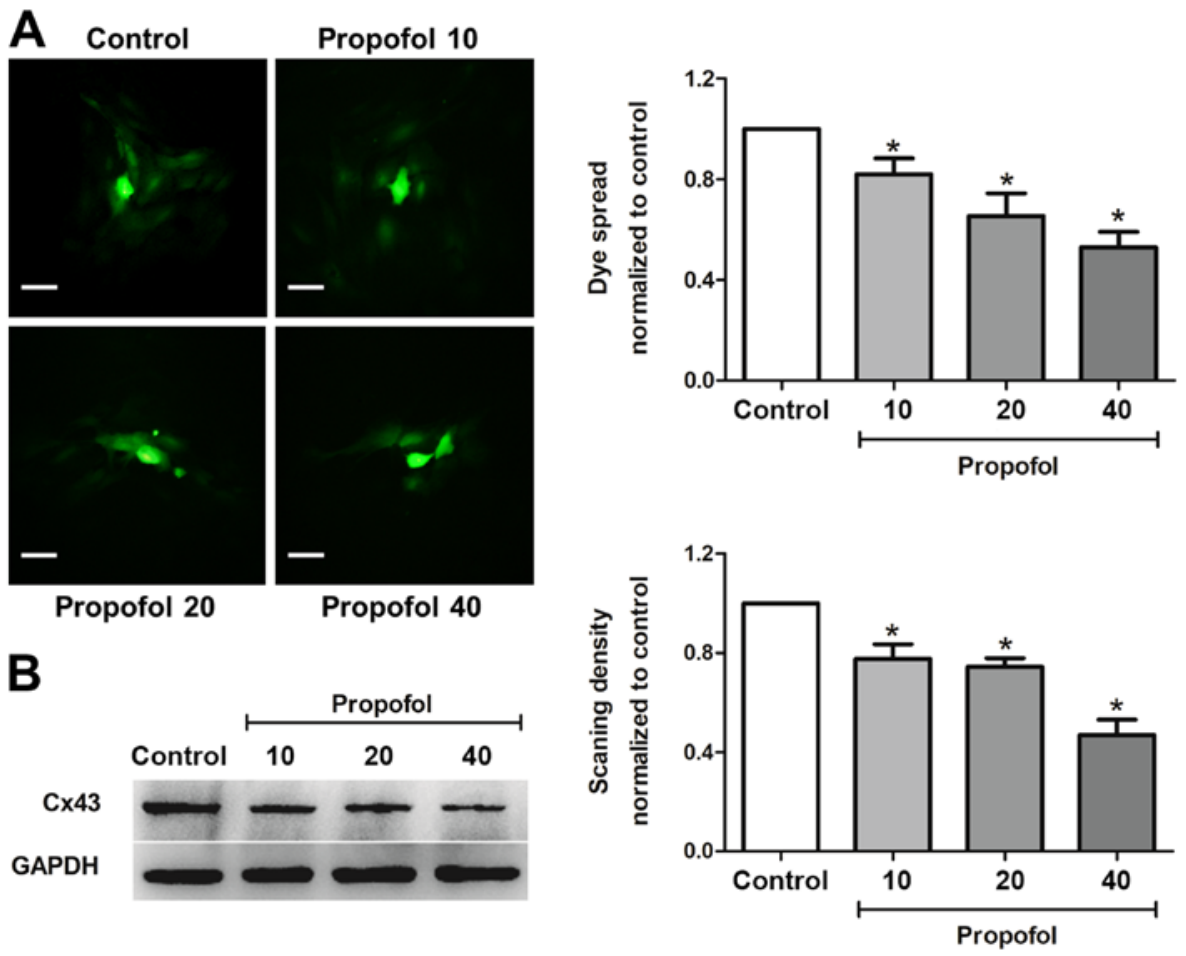

Figure 3. Effect of propofol on dye-coupling, gap junction function and $\mathrm{Cx} 43$ expression in astrocytes. (A) 'Parachute' assays were used to determine the gap junction function of astrocytes. (B) Cx43 expression was determined by western blot analysis. Values are presented as the mean \pm SEM of three independent experiments. ${ }^{*} \mathrm{P}<0.05$ vs. control group. $\mathrm{Cx} 43$, connexin 43.

were more sensitive to OGD/R injury. By contrast, the $\mathrm{Cx} 43$ KD group, with lower Cx43 expression, exhibited higher cell viability rates and lower apoptotic rates compared with the normal group after OGD/R injury, which further demonstrated that $\mathrm{Cx} 43$ can aggravate OGD/R-induced cell injury (Fig. 4C-E). Furthermore, propofol $(40 \mu \mathrm{M})$ diminished the OGD/R-induced cell injury in the normal and Cx43 OE groups, but not in the $\mathrm{Cx} 43 \mathrm{KD}$ group. These data indicated that $\mathrm{Cx} 43$ served an important role in propofol-suppressed OGD/R cell injury. Overall, the data demonstrated that propofol exerted neuroprotective effects against OGD/R cell injury by targeting $\mathrm{Cx} 43$ and inhibiting gap junction function.

\section{Discussion}

The present study revealed that GJIC serves an important role in OGD/R-induced astrocyte injury. When GJIC was inhibited by $\mathrm{CBX}$, cell damage was alleviated. Furthermore, propofol, at its clinically relevant concentrations, protected astrocytes from OGD/R-induced cell death via the inhibition of GJIC and $\mathrm{Cx} 43$ expression. This conclusion was supported by the following findings: i) Propofol inhibited OGD/R-induced apoptosis in astrocytes; ii) propofol markedly suppressed gap junction function and expression levels of $\mathrm{Cx} 43$ in astrocytes (Fig. 3A); and iii) $\mathrm{Cx} 43 \mathrm{OE}$ increased OGD/R-induced cell death and apoptosis, whereas propofol treatment had the opposite effect. On the contrary, in Cx43 KD cells, propofol exhibited only slight neuroprotective effects (Fig. 4).

Stroke is one of the leading causes of mortality and disability worldwide (1). Current treatments for stroke have limited benefits (2). In past decades, researchers have mainly focused on neurons to treat hypoxic-ischemic injury (24).
Previously, astrocytes, the most abundant cell type in the central nervous system, have been reported to be another potential target for stroke therapy (25). It has been reported that impaired astrocytes in stroke could stimulate several important apoptotic signaling pathways via gap junction communication, such as cellular $\mathrm{Ca}^{2+}$ overload, to exacerbate neuronal death (26). Accordingly, alleviating astrocyte injury could be an important therapeutic direction to protect neurons from stroke. In vitro $\mathrm{OGD} / \mathrm{R}$ astrocyte models have been widely used to mimic $H / R$ injury that occurs in stroke. The results of the present study demonstrated that OGD/R lead to astrocyte injury. Compared with the control group, astrocytes treated with OGD/R exhibited lower cell viability, a higher LDH leakage rate and a higher apoptotic rate. This result was consistent with previous studies $(27,28)$, which suggests that the OGD/R model used in the present study successfully mimicked hypoxic-ischemic brain injury. Propofol is a widely used intravenous sedative and anesthetic agent for both short-term anesthesia and longer-term sedation (12). Propofol has been reported to exert neuroprotective effects on cerebral ischemia-reperfusion injury $(20,29)$. In the present study, propofol, at its clinically relevant concentrations (10-40 $\mu \mathrm{M})$, dose-dependently decreased OGD/R-induced cell injury via inhibition of gap junction function. Gap junctions are important connections in direct cell-to-cell communication that serve essential roles in the pathogenesis of ischemic brain injury (30). Previous studies have demonstrated that both beneficial and harmful substances may pass through gap junctions to affect stroke in opposite ways $(25,31-33)$. The present results demonstrated that when GJIC was inhibited by CBX, OGD/R-induced cell injury was decreased, including the upregulation of 
A
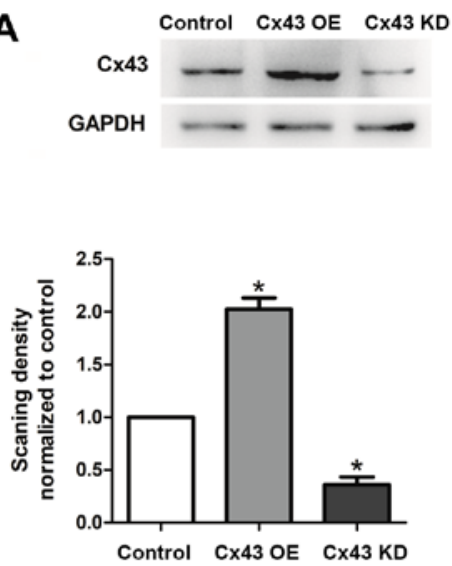

C

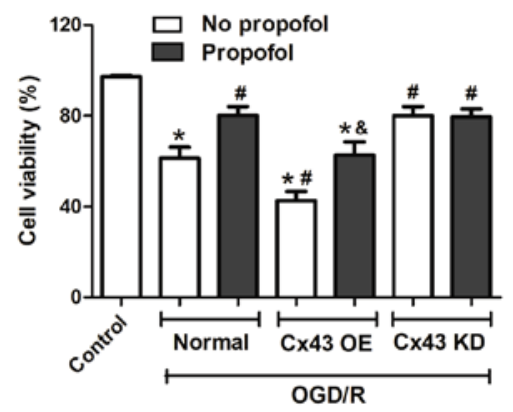

B
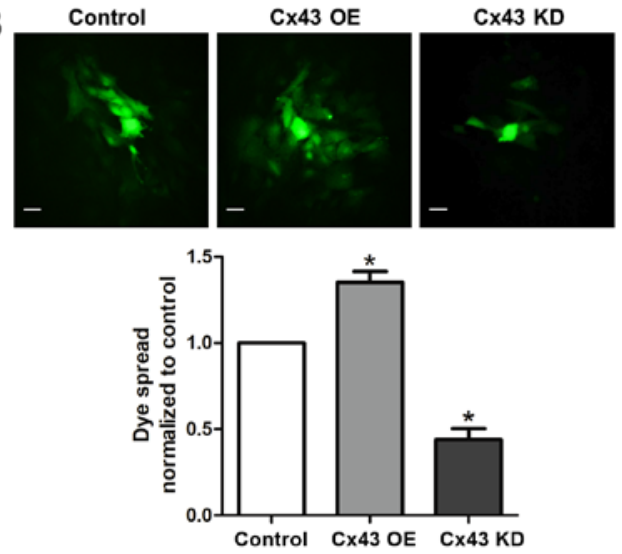

D

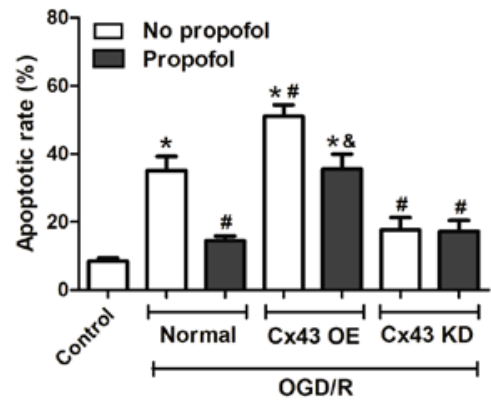

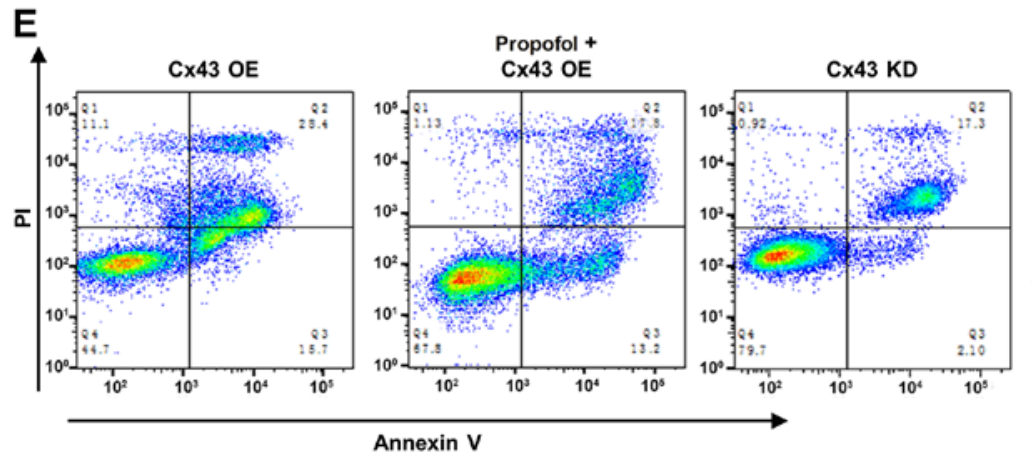

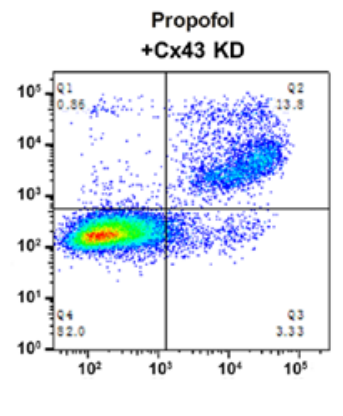

Figure 4. Effect of propofol on OGD/R-induced cell injury in Cx43 KD and Cx43 OE astrocytes. (A) Cx43 expression level and (B) gap junction function was upregulated in the $\mathrm{Cx} 43 \mathrm{OE}$ group, and downregulated in the $\mathrm{Cx} 43 \mathrm{KD}$ group compared with the control group (scale bar, $20 \mu \mathrm{m}$ ). (C) Cell viability and (D) apoptotic rate. (E) Representative flow cytometry images. Values are presented as the mean \pm SEM from three independent experiments. ${ }^{*}<<0.05$ vs. control group; ${ }^{\prime} \mathrm{P}<0.05$ vs. Normal + no propofol group; ${ }^{\circledR} \mathrm{P}<0.05$ vs. Cx43 OE + no propofol group. Cx43, connexin 4; KD, knockdown; OGD/R, oxygen-glucose deprivation and re-oxygenation; $\mathrm{OE}$, overexpression; PI, propidium iodide.

cell viability, and downregulation of LDH leakage and apoptotic rates. These results indicated that GJIC exacerbated OGD/R-induced cell injury in primary cultured astrocytes, which is consistent with a study by Orrenius et al (26), which reported that impaired astrocytes in stroke could stimulate several important apoptotic pathways via gap junction communication. Gap junction channels are composed of $\mathrm{Cx}$ proteins, of which $\mathrm{Cx} 43$ is one of the most abundant gap junction proteins in astrocytes (34). However, the role of $\mathrm{Cx} 43$ in stroke remains controversial. It has been reported that $\mathrm{Cx} 43$ aggravates ischemia-induced cell damage by spreading death signals to neighboring cells $(10,11,30)$. The present study found that propofol decreased the protein expression levels of $\mathrm{Cx} 43$, thereby protecting astrocytes from OGD/R-induced cell injury.
In the future, it would be noteworthy to investigate the mechanism by which propofol regulates the protein expression levels of $\mathrm{Cx} 43$. One hypothesis is that propofol treatment may trigger increased Cx43 degradation. Autophagy is a conserved cellular process to degrade unnecessary proteins. It has been reported that propofol treatment induces cellular protective autophagy in COS-7 cells under hypoxic conditions (35), therefore it would be useful to study whether propofol induces autophagy to degrade $\mathrm{Cx} 43$ in injured astrocytes. Another possibility is that propofol treatment suppresses the translation of $\mathrm{Cx} 43$. MicroRNAs are a class of non-coding RNAs $\sim 22$ nucleotides in length that inhibit gene expression; a series of studies have highlighted the roles of propofol in regulating the amount of various microRNAs in astrocytes (36-38). A recent study has found that mirocRNA-206 
targets the 3'untranslated regions of $\mathrm{Cx} 43$ in vascular smooth muscle cells (39). So, it would also be of note to investigate whether propofol downregulates the expression levels of $\mathrm{Cx} 43$ via regulation of microRNAs in the future.

In conclusion, the present study demonstrated that propofol reduced OGD/R-induced cell injury in astrocytes by inhibiting gap junction activity via downregulation of $\mathrm{Cx} 43$ protein expression. Therefore, the present study provides novel insights into the mechanism underlying the protective effect of propofol in astrocytes following $\mathrm{H} / \mathrm{R}$ injury.

\section{Acknowledgements}

Not applicable.

\section{Funding}

No funding was received.

\section{Availability of data and materials}

The datasets used and/or analyzed during the current study are available from the corresponding author on reasonable request.

\section{Authors' contributions}

YF, SZ and XW designed the experiments. YF, SZ, JW and $Y Z$ conducted experiments and data analysis. YF and SZ wrote the manuscript, and XW edited and approved the manuscript. All authors read and approved the final manuscript.

\section{Ethics approval and consent to participate}

The animal experiment protocol was reviewed and approved by the Institutional Animal Care and Use Committee of Sun Yat-sen University (Guangzhou, China).

\section{Patient consent for publication}

Not applicable.

\section{Competing interests}

The authors declare that they have no competing interests.

\section{References}

1. Majid A: Neuroprotection in stroke: Past, present, and future. ISRN Neurol 2014: 515716, 2014.

2. Yang G, Wang Y, Zeng Y, Gao GF, Liang X, Zhou M, Wan X, Yu S, Jiang Y, Naghavi M, et al: Rapid health transition in China, 1990-2010: Findings from the global burden of disease study 2010. Lancet 381: 1987-2015, 2013.

3. Rossi DJ, Brady JD and Mohr C: Astrocyte metabolism and signaling during brain ischemia. Nat Neurosci 10: 1377-1386, 2007.

4. Nedergaard M and Dirnagl U: Role of glial cells in cerebral ischemia. Glia 50: 281-286, 2005.

5. Harris AL: Connexin channel permeability to cytoplasmic molecules. Prog Biophys Mol Biol 94: 120-143, 2007.

6. Li X, Zhao H, Tan X, Kostrzewa RM, Du G, Chen Y, Zhu J, Miao Z, Yu H, Kong J and Xu X: Inhibition of connexin43 improves functional recovery after ischemic brain injury in neonatal rats. Glia 63: 1553-1567, 2015.
7. Contreras JE, Sánchez HA, Véliz LP, Bukauskas FF, Bennett MV and Sáez JC: Role of connexin-based gap junction channels and hemichannels in ischemia-induced cell death in nervous tissue. Brain Res Brain Res Rev 47: 290-303, 2004.

8. Sun JB, Li Y, Cai YF, Huang Y, Liu S, Yeung PK, Deng MZ, Sun GS, Zilundu PL, Hu QS, et al: Scutellarin protects oxygen/glucose-deprived astrocytes and reduces focal cerebral ischemic injury. Neural Regen Res 13: 1396-1407, 2018.

9. Siushansian R, Bechberger JF, Cechetto DF, Hachinski VC and Naus CC: Connexin43 null mutation increases infarct size after stroke. J Comp Neurol 440: 387-394, 2001.

10. Frantseva MV, Kokarovtseva L, Naus CG, Carlen PL, MacFabe D and Perez Velazquez JL: Specific gap junctions enhance the neuronal vulnerability to brain traumatic injury. J Neurosci 22 : 644-653, 2002.

11. Wasielewski B, Jensen A, Roth-Härer A, Dermietzel R and Meier C: Neuroglial activation and Cx43 expression are reduced upon transplantation of human umbilical cord blood cells after perinatal hypoxic-ischemic injury. Brain Res 1487: 39-53, 2012.

12. Deegan RJ: Propofol: A review of the pharmacology and applications of an intravenous anesthetic agent. Am J Med Sci 304: 45-49, 1992.

13. Ergün R, Akdemir G, Sen S, Tasçi A and Ergüngör F: Neuroprotective effects of propofol following global cerebral ischemia in rats. Neurosurg Rev 25: 95-98, 2002.

14. Bayona NA, Gelb AW, Jiang Z, Wilson JX, Urquhart BL and Cechetto DF: Propofol neuroprotection in cerebral ischemia and its effects on low-molecular-weight antioxidants and skilled motor tasks. Anesthesiology 100: 1151-1159, 2004.

15. Rossaint J, Rossaint R, Weis J, Fries M, Rex S and Coburn M Propofol: Neuroprotection in an in vitro model of traumatic brain injury. Crit Care 13: R61, 2009.

16. Huang Y, Zitta K, Bein B, Scholz J, Steinfath M and Albrecht M: Effect of propofol on hypoxia re-oxygenation induced neuronal cell damage in vitro*. Anaesthesia 68: 31-39, 2013.

17. Yamamoto N, Sobue K, Miyachi T, Inagaki M, Miura Y, Katsuya $\mathrm{H}$ and Asai K: Differential regulation of aquaporin expression in astrocytes by protein kinase C. Brain Res Mol Brain Res 95: 110-116, 2001

18. Schildge S, Bohrer C, Beck K and Schachtrup C: Isolation and culture of mouse cortical astrocytes. J Vis Exp 50079, 2013

19. Song MB, Yu XJ, Cui X, Zhu GX, Zhao G, Chen JF and Huang L: Blockade of connexin 43 hemichannels reduces neointima formation after vascular injury by inhibiting proliferation and phenotypic modulation of smooth muscle cells. Exp Biol Med (Maywood) 234: 1192-1200, 2009.

20. Sun B, Ou H, Ren F, Huan Y, Zhong T, Gao M and Cai H: Propofol inhibited autophagy through $\mathrm{Ca}(2+) / \mathrm{CaMKK} \beta / \mathrm{AMPK} / \mathrm{mTOR}$ pathway in OGD/R-induced neuron injury. Mol Med 24: 58 , 2018.

21. Tao L and Harris AL: 2-aminoethoxydiphenyl borate directly inhibits channels composed of connexin26 and/or connexin32. Mol Pharmacol 71: 570-579, 2007.

22. Koreen IV, Elsayed WA, Liu YJ and Harris AL: Tetracycline-regulated expression enables purification and functional analysis of recombinant connexin channels from mammalian cells. Biochem J 383: 111-119, 2004.

23. Zhao Y, Liu B, Wang Q, Yuan D, Yang Y, Hong X, Wang X and Tao L: Propofol depresses the cytotoxicity of X-ray irradiation through inhibition of gap junctions. Anesth Analg 112: 1088-1095, 2011.

24. Busl KM and Greer DM: Hypoxic-ischemic brain injury: Pathophysiology, neuropathology and mechanisms. NeuroRehabilitation 26: 5-13, 2010.

25. Liu Z and Chopp M: Astrocytes, therapeutic targets for neuroprotection and neurorestoration in ischemic stroke. Prog Neurobiol 144: 103-120, 2016.

26. Orrenius S, Zhivotovsky B and Nicotera P: Regulation of cell death: The calcium-apoptosis link. Nat Rev Mol Cell Biol 4: 552-565, 2003.

27. Li CY, Li X, Liu SF, Qu WS, Wang W and Tian DS: Inhibition of mTOR pathway restrains astrocyte proliferation, migration and production of inflammatory mediators after oxygen-glucose deprivation and reoxygenation. Neurochem Int 83-84: 9-18, 2015.

28. Wang N, Zhang Y, Wu L, Wang Y, Cao Y, He L, Li X and Zhao J: Puerarin protected the brain from cerebral ischemia injury via astrocyte apoptosis inhibition. Neuropharmacology 79: 282-289, 2014. 
29. Wang H, Zheng S, Liu M, Jia C, Wang S, Wang X, Xue S and Guo Y: The effect of propofol on mitochondrial fission during oxygen-glucose deprivation and reperfusion injury in rat hippocampal neurons. PLoS One 11: e0165052, 2016.

30. Deng ZH, Liao J, Zhang JY, Liang C, Song CH, Han M, Wang LH, Xue $\mathrm{H}$, Zhang K, Zabeau L, et al: Inhibition of the connexin 43 elevation may be involved in the neuroprotective activity of leptin against brain ischemic injury. Cell Mol Neurobiol 34 871-879, 2014

31. Wallraff A, Köhling R, Heinemann U, Theis M, Willecke K and Steinhäuser C: The impact of astrocytic gap junctional coupling on potassium buffering in the hippocampus J Neurosci 26: 5438-5447, 2006.

32. Nodin C, Nilsson $\mathrm{M}$ and Blomstrand F: Gap junction blockage limits intercellular spreading of astrocytic apoptosis induced by metabolic depression. J Neurochem 94: 1111-1123, 2005.

33. Perez Velazquez JL, Frantseva MV and Naus CC: Gap junctions and neuronal injury: Protectants or executioners? Neuroscientist 9: 5-9, 2003

34. Rouach N, Avignone E, Meme W, Koulakoff A, Venance L, Blomstrand $\mathrm{F}$ and Giaume C: Gap junctions and connexin expression in the normal and pathological central nervous system. Biol Cell 94: 457-475, 2002.
35. Yoon JY, Baek CW, Kim EJ, Park BS, Yu SB, Yoon JU and Kim EN: Propofol protects against oxidative-stress-induced COS-7 cell apoptosis by inducing autophagy. J Dent Anesth Pain Med 17: 37-46, 2017

36. Lu Y, Jian M, Xiong W and Han R: Effects of propofol on miR-181a and $\mathrm{Bcl}-2$ expression in glucose deprivation cultured astrocytes. Zhonghua Yi Xue Za Zhi 94: 3020-3023, 2014 (In Chinese).

37. Sun WC, Liang ZD and Pei L: Propofol-induced rno-miR-665 targets BCL2L1 and influences apoptosis in rodent developing hippocampal astrocytes. Neurotoxicology 51: 87-95, 2015.

38. Sun W and Pei L: MicroRNA expression profiling of propofol-treated developing rat hippocampal astrocytes. DNA Cell Biol 34: 511-523, 2015.

39. Li H, Xiang Y, Fan LJ, Zhang XY, Li JP, Yu CX, Bao LY, Cao DS, Xing WB, Liao XH and Zhang TC: Myocardin inhibited the gap protein connexin 43 via promoted miR-206 to regulate vascular smooth muscle cell phenotypic switch. Gene 616: 22-30, 2017.

This work is licensed under a Creative Commons

Attribution-NonCommercial-NoDerivatives 4.0 International (CC BY-NC-ND 4.0) License. 